\title{
A Case of Multiple Piloleiomyoma on the Shoulder, which is Mistaken for Keloid
}

\author{
Syeo Young Wee, Jun Ho Park, \\ Hyun Gyo Jeong, \\ Chang Yong Choi
}

Department of Plastic and Reconstructive Surgery, Soonchunhyang Gumi Hospital, Soonchunhyang University College of Medicine, Gumi, Korea

This work was supported by the Soonchunhyang University Research Fund.

No potential conflict of interest relevant to this article was reported.

\begin{abstract}
A 30-year-old male presented with multiple tender erythematous nodules, varying in size from $0.5 \mathrm{~cm}$ to $2 \mathrm{~cm}$ on the right shoulder of the body. He gave a history of pain associated with these lesions, especially on exposure to cold and touch. These lesions showed multi-segmental distribution; each began as a papule, which gradually increased both in size and number to form a nodule. The lesions, which were misdiagnosed as keloids at another hospital, were managed with numerous intralesional steroid injections. This treatment did not improve the symptoms. Based on the history and cutaneous findings during the physical examination, we considered a diagnosis of a benign tumor. Among the lesions, some of the larger ones were completely excised, followed by excision biopsy. We then made a diagnosis of cutaneous leiomyoma, more specifically, piloleiomyoma. The excisional lesions were covered by skin grafts and closed by primary repair.
\end{abstract}

Keywords Piloleiomyoma, Leiomyoma, Keloid, Shoulder

\section{INTRODUCTION}

In the cases of a papule or nodule emerging from the skin at the shoulder, with signs of itching or pain among the Asian populations, we suspect the lesion to be a keloid. This is because children used to be inoculated with Bacillus Calmette-Guérin in the shoulder.

Itching and pain are sensations that are commonly felt by patients who have keloids. A previous study reported that $86 \%$ of the patients experienced itching, and $46 \%$ felt pain [1].

Leiomyoma, on the other hand, is a rare benign tumor that appears in either solitary or multiple patterns. However, cutaneous leiomyomas may look clinically similar to keloids and other benign cutaneous neoplasms, therefore a diagnosis can only be confirmed by examining the characteristic histology of the lesion.

We report the case of a patient who came to our hospital because

Received: Apr 9, 2015 Revised: May 27, 2015 Accepted: May 27, 2015

Correspondence: Chang Yong Choi Department of Plastic and

Reconstructive Surgery, Soonchunhyang University Gumi Hospital,

179 Gongdan-ro 1, Gumi 730-706, Korea. E-mail: ccysy@hanmail.net

Copyright @ 2015 The Korean Society for Aesthetic Plastic Surgery.

This is an Open Access article distributed under the terms of the Creative Commons Attribution Non-Commercial License (http://creativecommons.org/licenses/by-nc/3.0/) which permits unrestricted non-commercial use, distribution, and reproduction in any medium, provided the original work is properly cited. www.e-aaps.org his symptoms were not improving after treatment at another hospital, where he had been misdiagnosed as having a keloid.

\section{CASE}

A 30-year-old male presented with multiple tender erythematous nodules, varying in size from $0.5 \mathrm{~cm}$ to $2.0 \mathrm{~cm}$, on the right shoulder of his body; the nodules had been present for about 5 years. These lesions showed a multi-segmental distribution and were isolated to the skin of the right shoulder. Each lesion had begun as a papule that gradually increased in size and number to form a nodule (Fig. 1).

The patient described a history of pain associated with these lesions, especially on exposure to cold and slight touch. The pain disrupted his daily life and intensified at night. His symptoms also included an itching sensation.

His condition had been managed with numerous intralesional steroid injections, because the lesions had been misdiagnosed as keloids at another hospital. Since he was disturbed by the pain and itching, he was treated more than 10 times using triamcinolone injections at the original hospital. However, the patient's symptoms had not improved. During a cutaneous examination, the lesions were found to contain multiple small masses that did not appear to be increasing rapidly in size. The surface of the masses was also 
smooth rather than rough. Based on his history and the results of the cutaneous examination, we considered a diagnosis of a different type of a benign tumor. We performed a surgical excision of some larger masses and took a biopsy (Fig. 2A). The excised lesions were covered by a skin graft and closed by primary repair (Fig. 2B). The remnants of the lesions were preserved until the diagnosis was

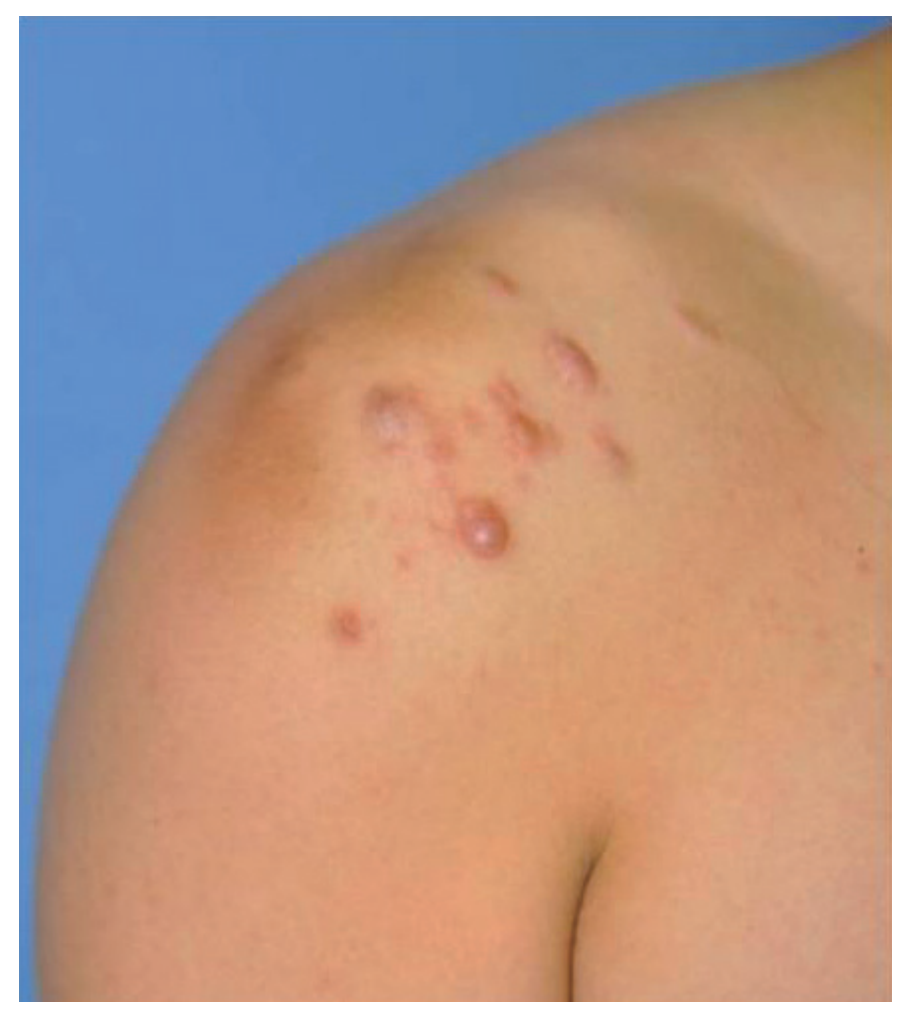

Fig. 1. The patient had multiple masses on the shoulder.

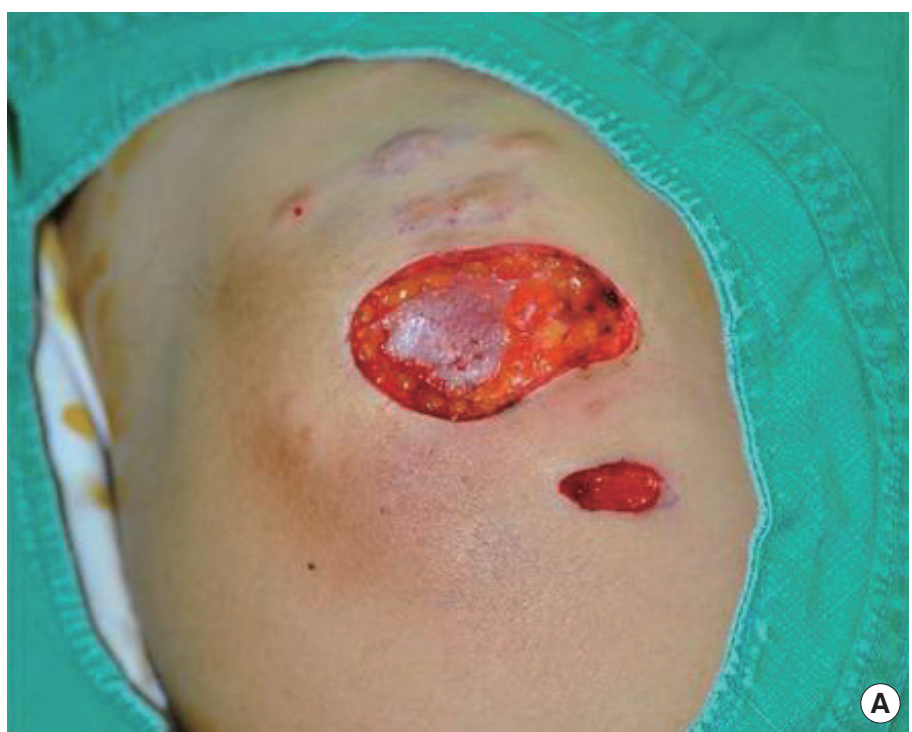

confirmed.

Histopathology revealed interlacing and whorled patterns of smooth muscle, collagen fibers, and variable amounts of lymphocyte and mast cell infiltration occupying the entire reticular dermis (Fig. 3A). In addition, high-power microscopy showed no mitoses or atypia (Fig. 3B). We were able to find mature smooth muscle cells by immunohistochemical staining for smooth muscle actin (Fig. 4A and B).

We then made a diagnosis of cutaneous leiomyoma, more specifically, piloleiomyoma. We thus planned its surgical removal and assessment of the site every month for recurrence.

\section{DISCUSSION}

Leiomyoma originates from smooth muscles. It can be classified as piloleiomyoma (pilar leiomyoma) from arrector pili muscles, angioleiomyoma from blood vessels, and genital leiomyoma from the smooth muscles of the scrotum, labia majora, and nipple, depending on the emerging area [2]. Cutaneous leiomyoma is a rare benign tumor of the skin, and it usually appears in solitary form. Piloleiomyoma is among the most common variants of cutaneous leiomyoma [3]. It typically presents as firm, reddish-brown or skincolored nodules, generally over the face, neck, trunk, and extremities. Its size may gradually increase and new lesions may arise [4,5]. Piloleiomyoma can present in a solitary or a grouped pattern. Multiple patterns are about 1.2 times more frequent than solitary patterns. It can emerge on the limbs in both solitary and multiple patterns but solitary is more common [6]. The diameter of the mass of a solitary pattern is smaller than $15 \mathrm{~mm}$, which makes a diagnosis difficult to confirm [7].

Patients usually complain of hypersensitivity to pressure, as well

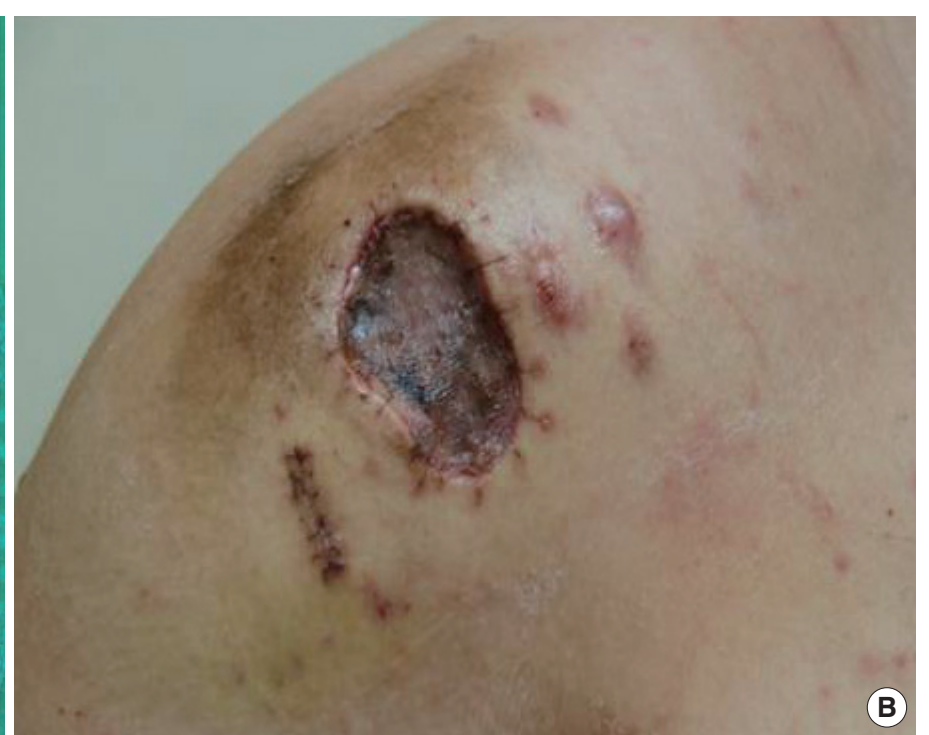

Fig. 2. (A) The masses were excised. (B) The excisional lesions were covered by skin graft and closed by primary repair. 

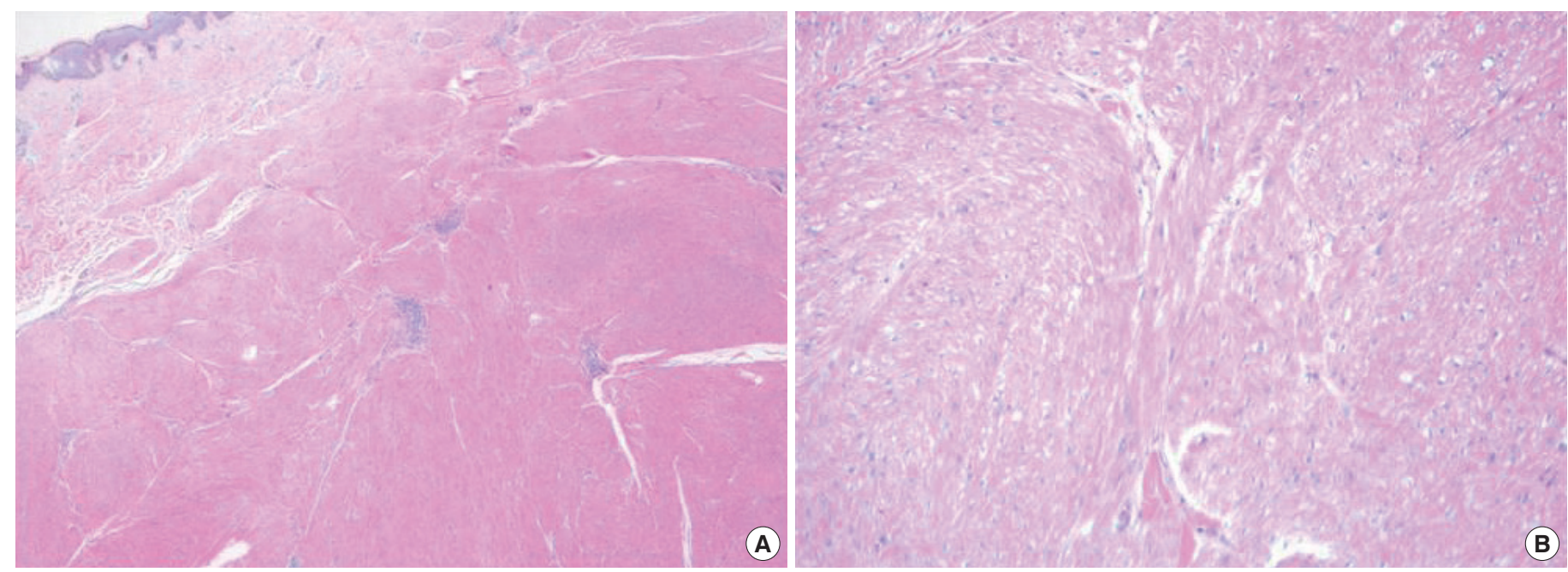

Fig. 3. (A) The pathologic finding of the masses (hematoxylin-eosin $[H \& E] \times 40)$. (B) The pathologic finding of the masses $(H \& E, \times 100)$.
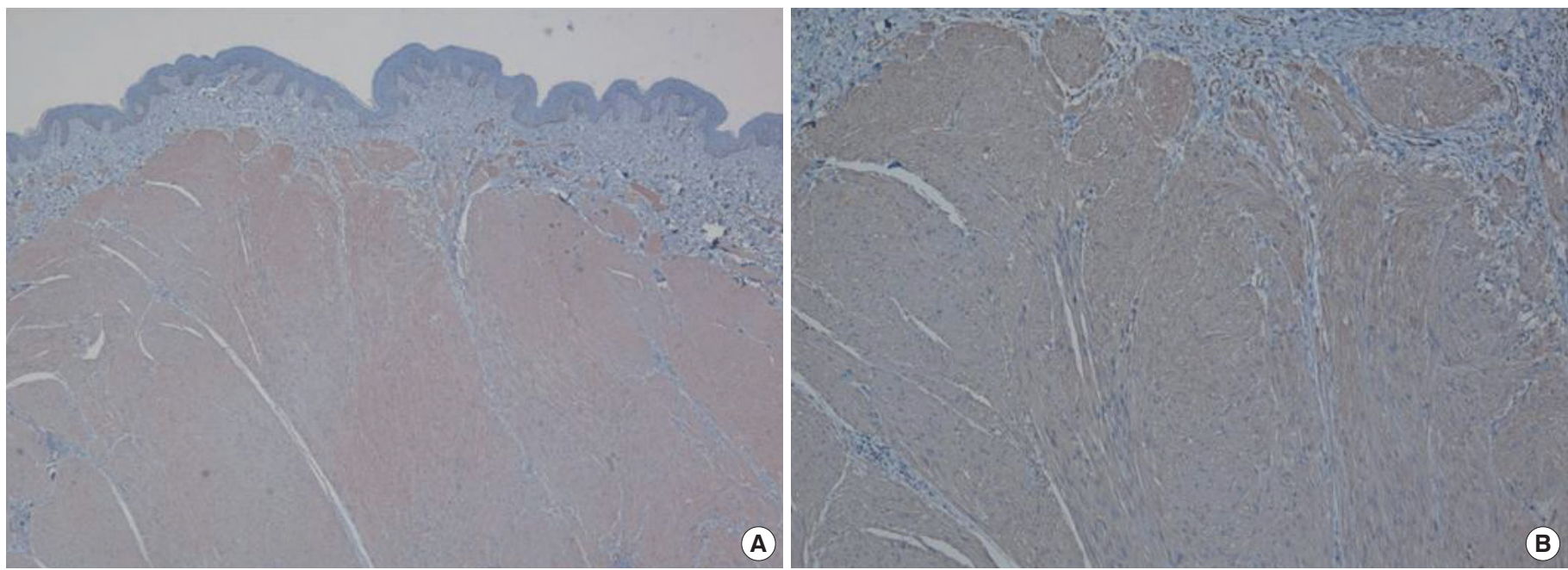

Fig. 4. (A) The pathologic finding of the masses (smooth muscle actin immunohistochmistry [IHC] stain, $\times 40$ ). (B) The pathologic finding of the masses (smooth muscle actin IHC stain, $\times 100$ ).

as to light touch and cold temperature, which may be spontaneous or induced by trauma. The pathognomonic symptom is the pain that is provoked by light touch. This hypersensitivity and pain may be induced by the localized compression of the tumor itself, which contains the cutaneous nerve [8], by contraction of the muscle fiber of the tumor [9], or by infiltration of tumor mast cells. However, not all of the causes of this hypersensitivity have been clarified [7].

In our case, the piloleiomyoma appeared as a grouped, protruding pattern with symptoms of acute pain accompanied by pruritus. It is thought that the painful sensation is normally recognized as an itching sensation with the existence of central sensitization [10]. Because of this itching sensation, the lesion can be misdiagnosed as a keloid.

Surgical excision and skin grafting are the gold standard for treat- ing cutaneous leiomyoma 4, because cutaneous leiomyomas grow gradually and new lesions emerge. The goal of the surgical procedure is to remove the lesion and resect the entire margin, because the rate of recurrence is high from the first 6 weeks through 15 years after resection [11].

Some drugs, for example, nitroglycerin, nifedipine, phenoxybenzamine, and doxazosin, which are effective for inducing smooth muscle contraction, can help reduce pain. Calcium channel blockers, botulinum toxin type $\mathrm{A}$, and antidepressants have recently been used for treatment as well $[12,13]$. We did not use such drugs before the surgery because we could only diagnose the lesions after the biopsy. Some studies have shown that traimacinolone acetonide injections decrease the pain and size of the lesions [13]. However, in our case, the steroid injections that were administered after the patient was misdiagnosed with keloids were not effective, and 
he continued to experience symptoms.

As seen by histological examination, leiomyoma has no cytological atypia or pleomorphism in the dermal lesions under high-power microscopy. Smooth muscle cells have abundant eosinophilic cytoplasm, blunt-ended and elongated nuclei, and a perinuclear vacuole [8]. They also have low mitotic activity in comparison to leiomyosarcoma [7]. A diagnosis of leiomyoma can be confirmed through immunohistochemical staining to identify smooth muscle actin, consequently, for an accurate diagnosis, a biopsy of the lesion is required.

Therefore, if a patient complains of pain or another hyperesthetic sensitization and has patterned skin lesions that are gradually growing, especially if the patient has severe pain associated with that lesion, a diagnosis of cutaneous leiomyoma can be suspected.

\section{PATIENT CONSENT}

Patients provided written consent for the use of their images.

\section{REFERENCES}

1. Lee SS, Yosipovitch G, Chan YH, et al. Pruritus, pain, and small nerve fiber function in keloids: a controlled study. J Am Acad Dermatol 2004; 51:1002-6.

2. Calonje E. Soft-tissue tumours and tumour-like conditions. In: Burns T, Breathnach S, Cox N, et al., editors. Rook's textbook of dermatology. 8th ed. Oxford, UK: Wiley-Blackwell; 2010. p.2797-858.

3. Usmani N, Merchant W, Yung A. A case of cutaneous symplastic leio- myoma - a rare variant of cutaneous pilar leiomyoma. J Cutan Pathol 2008;35:329-31.

4. Holst VA, Junkins-Hopkins JM, Elenitsas R. Cutaneous smooth muscle neoplasms: clinical features, histologic findings, and treatment options. J Am Acad Dermatol 2002;46:477-90.

5. Smith CG, Glaser DA, Leonardi C. Zosteriform multiple leiomyomas. J Am Acad Dermatol 1998;38:272-3.

6. Mitra A, Gudgeon PW, Merchant W, et al. A case of diffuse pilar leiomyoma or acquired smooth muscle hamartoma? Clin Exp Dermatol 2009;34:e145-7.

7. Raj S, Calonje E, Kraus M, et al. Cutaneous pilar leiomyoma: clinicopathologic analysis of 53 lesions in 45 patients. Am J Dermatopathol 1997;19:2-9.

8. Matthews JH, Pichardo RO, Hitchcock MG, et al. Cutaneous leiomyoma with cytologic atypia, akin to uterine symplastic leiomyoma. Dermatol Surg 2004;30:1249-51.

9. Arfan ul B. Zosteriform cutaneous leiomyoma: a rare cutaneous neoplasm. J Coll Physicians Surg Pak 2013;23:586-7.

10. Schmelz M. Itch and pain. Neurosci Biobehav Rev 2010;34:171-6.

11. Emer JJ, Solomon S, Mercer SE. Reed's Syndrome: A Case of Multiple Cutaneous and Uterine Leiomyomas. J Clin Aesthet Dermatol 2011;4: 37-42.

12. Christenson LJ, Smith K, Arpey CJ. Treatment of multiple cutaneous leiomyomas with CO2 laser ablation. Dermatol Surg 2000;26:319-22.

13. Liu C, Tang ZH, Bei H, et al. Treatment of a patient with multiple cutaneous piloleiomyoma-related pain with a local injection of triamcinolone acetonide. Dermatology 2013;227:52-4. 